\title{
Judge the Extent of the Harm on Electric Cables Based on Optical Fiber Sensing Technology and Found a New Mechanism of Patrolling Electric Cables
}

\author{
Xianjin Yang ${ }^{1, *}$, Jingqing Ye ${ }^{1}$,Guangyuan Yao ${ }^{1}$, Junfeng Chi ${ }^{1}$, and Jiali Du ${ }^{2}$ \\ ${ }^{1}$ Hangzhou Electric Power Design Institute Co., Ltd., 310000 in Hangzhou, Zhejiang \\ ${ }^{2}$ Guangzhou beam Information Technology Co., Ltd., 510000 in Guangzhou, Guangdong
}

\begin{abstract}
The article comes up with a measure that uses fiber vibration sensing technology to avoid external intrusion harm on electric cables, dealing with the problem that buried cables are sometimes destroyed by constructions. This measure is based on dual Mach-Zehnder(M-Z) interference, and it makes use of power grids' fiber-optic cables laid in the cable trench - along with the electric cables - as sensors. These sensors could monitor vibration near to optical cables and trench, and recognize the events that vibrate the cable, and finally judge the extent of the harm caused by the events. The article also try to find out a more reliable and more efficient mechanism of patrolling electric cables, which is based on the abovementioned measure.
\end{abstract}

\section{Introduction}

With the huge power load pressure in cities, the scale of power cables for power grid construction has increased year by year. At the same time, in order to take into account the beauty of the city, most of the power cables in urban areas are buried in cable trenches. Due to its concealed characteristics, buried power cables are easily damaged by road construction and other external force intrusion events ${ }^{[1]}$.

At present, power units mainly rely on inspections of cables to prevent cables from being damaged by external forces. The main methods include manual inspections, communication with construction units, and mutual notifications among internal employees. None of these inspection methods have the effect of real-time fullrange coverage and protection. For long-distance cables, there are large gaps and blind spots for monitoring, which can easily make illegal construction become a fish in the net and damage the cable. In order to prevent cables from being damaged by external forces, current power units need a technology that can detect behaviors that threaten the safety of cables that can run around the clock without interruption, so as to provide early warning before the cables are actually damaged ${ }^{[2]}$.

Optical fiber sensing technology is a technology that directly uses optical fiber as a sensor to sense external temperature and vibration. Among them, optical fiber vibration sensing is a technology that can detect and warn of external force events that threaten cable safety. Since there are common communication optical cables laid in the same trench for buried cables, the optical fiber can be used as a vibration sensor required by optical fiber sensing technology to detect vibrations near the cable, which can early detect external force intrusion events that threaten the safety of the cable, such as excavation.

This article mainly studies the use of optical fiber vibration sensing technology, using the power communication optical cable laid with the buried cable to monitor the vibration of the buried cable trench where the optical cable is located, and judge whether the vibration event will endanger the safety of the cable and cable trench, and the research is based on this technology establishes a new way of cable operation and maintenance inspection.

\section{Optical fiber vibration sensing technology}

\subsection{Principles of optical fiber vibration sensing technology}

The principle of mainstream optical fiber vibration sensing technology is mainly divided into two categories: phase-sensitive optical time-domain reflection ( $\Phi$ OTDR) technology and dual Mach-Zehnder interference (M-Z interference) technology.

$\Phi$-OTDR technology ${ }^{[3]}$ uses the Rayleigh scattering phenomenon when light is transmitted in an optical fiber. When the optical signal is transmitted in the optical fiber, the scattered light that is transmitted backward due to Rayleigh scattering will change the phase due to the change of the fiber stress caused by the fiber vibration at the scattering point. The phase change causes the change of the scattered light power. The receiving end analyzes

* Corresponding author: yangxj669366@163.com 
the change of the light power over a period of time and finds the vibration of the optical fiber ${ }^{[4]}$. The disadvantage of $\Phi$-OTDR technology is that it can only analyze the amplitude and frequency of the vibration, but cannot determine the event that caused the vibration. At the same time, it is too sensitive to the external environment. If it is used to determine the degree of external force intrusion, its false alarm rate is very high ${ }^{[5]}$, and it lacks practical value.

The following focuses on the dual $\mathrm{M}-\mathrm{Z}$ interference technology.

\subsection{Double $M-Z$ interference technology}

(1) Principle of Double M-Z Interference Technology

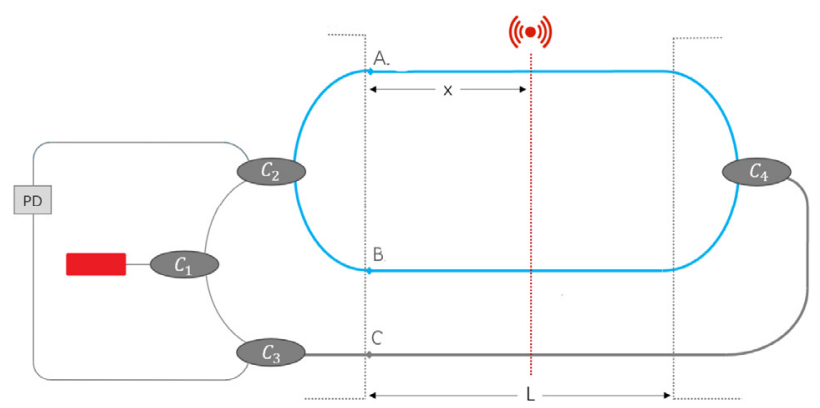

Fig.1. Schematic diagram of dual M-Z interference technology to detect fiber vibration.

The coherent light emitted by the laser is divided into two beams at the coupler $\mathrm{C}_{1}$ and goes to the couplers $\mathrm{C}_{2}$ and $\mathrm{C}_{3}$ respectively.

The coherent light reaching $C_{2}$ is divided into two beams again, which are respectively transmitted along the $\mathrm{A}$ and $\mathrm{B}$ sensing fibers from the start point to the end point. The two beams of light arrive at $\mathrm{C}_{4}$ to interfere, and the interfered light travels back to the starting point along the transmission fiber $\mathrm{C}$ for signal processing. The light path is ${ }^{[7]}$ :

$$
\text { Laser } \rightarrow \mathrm{C}_{1} \rightarrow \mathrm{C}_{2} \rightarrow \mathrm{C}_{3} \rightarrow \mathrm{C}_{4} \rightarrow \mathrm{PD}
$$

The coherent light that goes directly from $C_{1}$ to $C_{3}$ reaches the end point along the transmission fiber $\mathrm{C}$, and is divided into two beams at $\mathrm{C}_{4}$, and travels along the $\mathrm{A}$ and $\mathrm{B}$ sensing fibers from the end point to the starting point, and reaches $\mathrm{C}_{2}$ for interference. The interference light is directly sent to the signal processing. The light path is:

$$
\text { Laser } \rightarrow \mathrm{C}_{3} \rightarrow \mathrm{C}_{4} \rightarrow \mathrm{C}_{2} \rightarrow \mathrm{C}_{1} \rightarrow \mathrm{PD}
$$

The whole system include two $\mathrm{M}-\mathrm{Z}$ interferometers with opposite optical paths, and the optical paths are as described in formula (1) and formula (2) respectively.

When the sensing fibers $\mathrm{A}$ and $\mathrm{B}$ are disturbed, the phase of the transmitted light is affected, and the light intensity waveform after the interference of the two beams transmitted in the same direction will change [8]. The waveform example after receiving and signal processing through PD is shown in Figure 2: a. Output waveform when no disturbance is applied to the optical fiber (still affected by natural background noise):

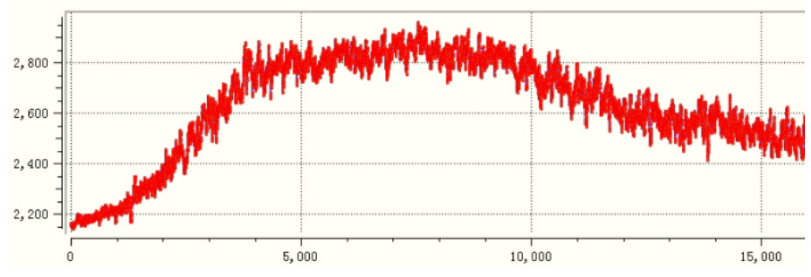

Fig.2. Output waveform when no disturbance is applied in double M-Z interference technology.

b. Output waveform when disturbance is applied to the fiber:

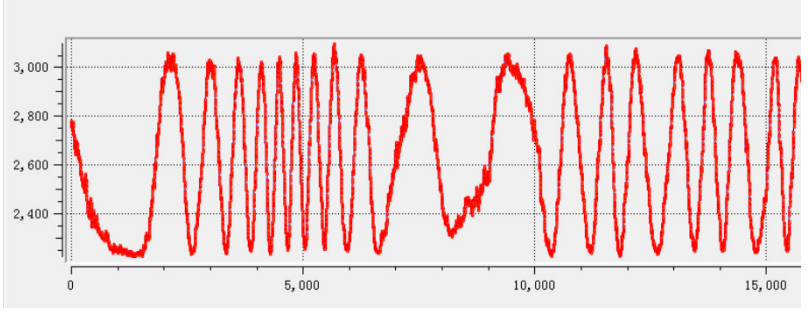

Fig.3. Output waveform when there is disturbance applied in double $\mathrm{M}-\mathrm{Z}$ interference technology.

It can be seen from Figures 2 and 3 that the vibration received by the optical fiber can be judged by the change of the output waveform.

\section{( 2 ) Positioning principle of double $\mathrm{M}-\mathrm{Z}$ interference} technology

The classic $M-Z$ interferometer cannot achieve positioning, so dual $\mathrm{M}-\mathrm{Z}$ interference technology is used, that is, the most important significance of dual $M-Z$ interference is to achieve positioning ${ }^{[9]}$.

Suppose the distance between the start point and the end point is $\mathrm{L}$, and a point on the optical fiber at a distance $\mathrm{x}$ from the start point produces vibration. Suppose the time taken for the transmitted light in the sensing fiber to reach the starting point from the vibration source is $\mathrm{t}$.

The time consumption of the optical path shown in formula (1) is:

$$
t_{1}=\frac{(L-x)+L}{c / n}
$$

$\mathrm{c}$ is the speed of light in vacuum, $\mathrm{n}$ is the refractive index of the fiber.

The time consumption of the optical path shown in formula (2) is:

$$
t_{2}=\frac{x}{c / n}
$$

$\mathrm{c}$ is the speed of light in vacuum, $\mathrm{n}$ is the refractive index of the fiber.

The time difference between the two lights is: 


$$
\tau=t_{1}-t_{2}=\frac{2 \cdot(L-x)}{c / n}
$$

From equation (5):

$$
x=L-\frac{c \cdot \tau}{2 n}
$$

Therefore, by detecting the time difference between the two paths of light reaching the starting point, the distance $\mathrm{x}$ of the vibration source can be calculated, which is a simple principle of double $\mathrm{M}-\mathrm{Z}$ interference positioning ${ }^{[10]}$.

\section{Use optical fiber vibration sensing technology to determine the degree of damage to cables by external forces}

\subsection{Distributed optical fiber vibration sensor based on double $M-Z$ interference technology}

Based on the principle of using dual $\mathrm{M}-\mathrm{Z}$ interference technology to detect optical fiber vibration, a dual $M-Z$ interference distributed optical fiber vibration sensor using dual light sources (referred to as optical fiber vibration sensor) is proposed here. The system schematic diagram is shown in Figure 4:

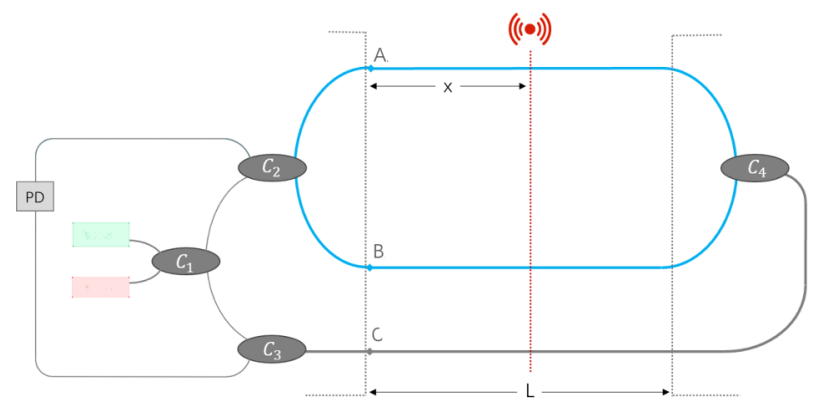

Fig.4. Dual M-Z interference distributed optical fiber vibration sensor with dual light sources.

The dual MZ interference distributed optical fiber vibration sensor with dual light sources uses two lasers to emit coherent light with slightly different frequencies from each other ${ }^{[11]}$, and the two coherent lights are first mixed into a small difference frequency mixed light ${ }^{[12]}$, The optical path of the mixed light is the same as formula (1) and formula (2). The positioning technology is the same as equation (6).

The function of using the small difference frequency mixing light is to make the waveform of the interference light easier to analyze and make the positioning of the vibration source more accurate and stable.

\subsection{Use distributed optical fiber vibration sensor to determine vibration events}

In order to study the ability of the distributed optical fiber vibration sensor to determine vibration events, we connected the optical fiber vibration sensor to the power communication optical cable laid in the cable trench, and used two optical fibers in the communication optical cable as the sensing optical fiber ( There is also a transmission optical fiber) to monitor vibration events occurring on the land along the optical cable.

The output signal waveform of the optical fiber vibration sensor after processing the interference light (when the optical fiber has no external vibration) is shown in Figure 5:
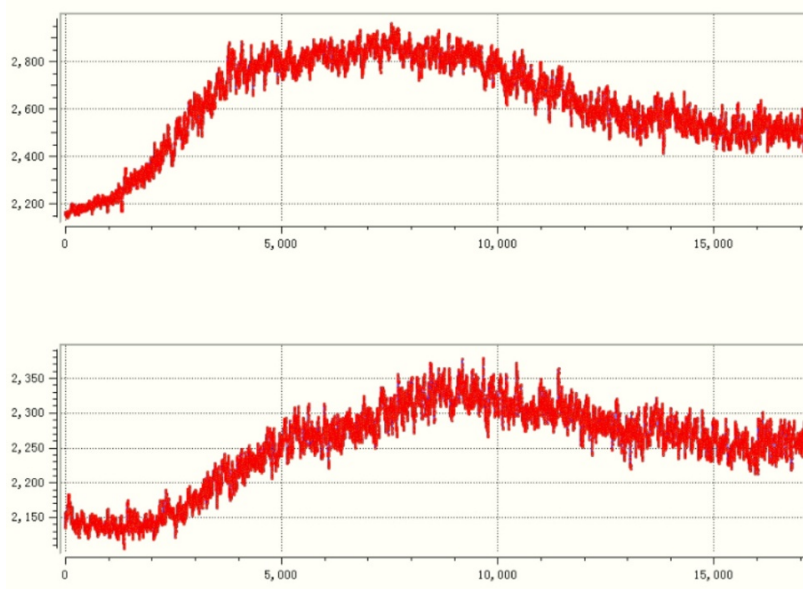

Fig.5. Output waveform without external vibration.

The upper and lower waveforms are the output waveforms of the light transmitted in the direction of the starting point $\rightarrow$ the end point and the direction of the end $\rightarrow$ starting point on the sensing fiber respectively.

The sensing fiber is affected by natural factors such as background noise and normal ground vibration events. Even if the sensing fiber is not disturbed, the waveform will have ups and downs and glitches. But the ups and downs are relatively gentle.

When vibration is applied to the sensing fiber, the output waveform will change, as shown in Figure 6 and Figure 7.

Comparing Figure 6 and Figure 7, we can find that the output waveform when a person directly touches the sensing fiber is a relatively regular periodic oscillation waveform, and the frequency of oscillation is very high; while the sensing fiber is close to the ground when there is an excavator on the ground. The output waveform of is an irregularly oscillating waveform, and its oscillation frequency is relatively low. Humans or machines can distinguish events such as normal noise, direct touch of the optical fiber, and road construction near the optical fiber. 


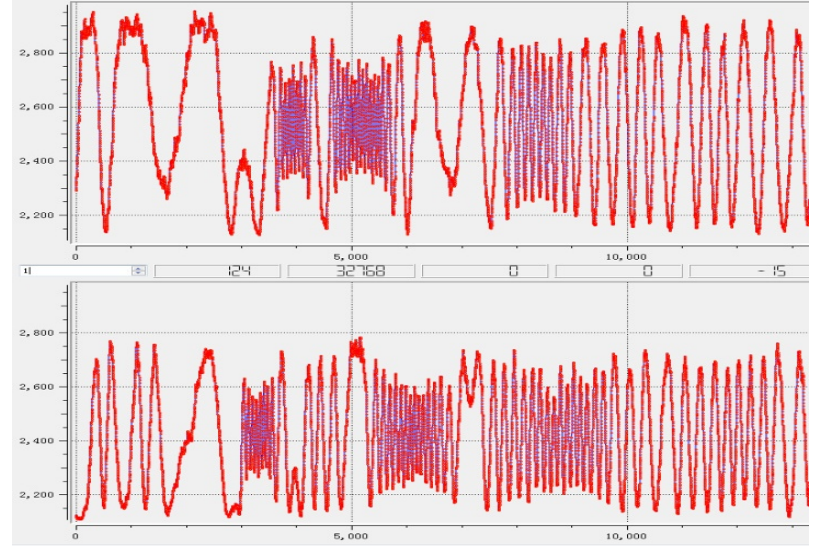

Fig.6. Output waveform when a person directly touches the sensing fiber.

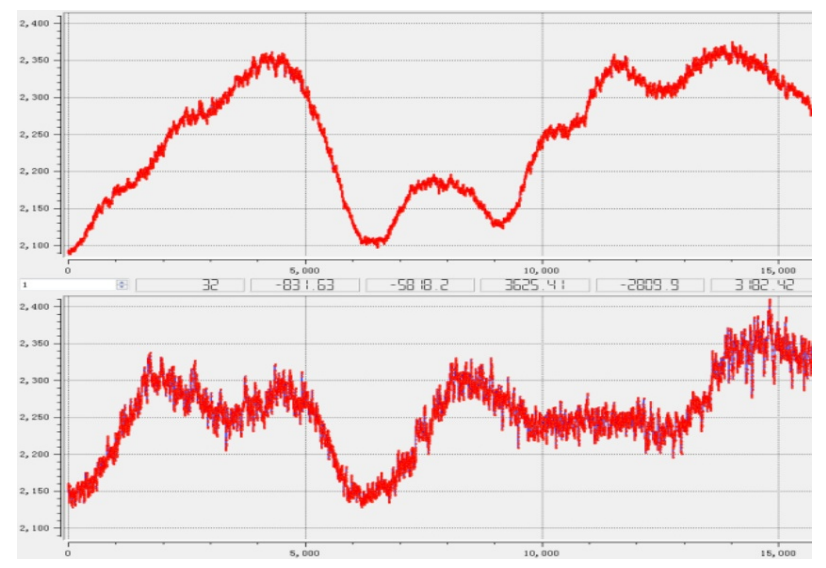

Fig.7. The output waveform when the excavator is near the ground of the sensing fiber.

It can be seen that if it is affected by vibration events such as hand touch or excavator construction nearby (and such events may often damage optical cables and cable trenches), the output waveform will have obvious characteristics, and the output waveform without the influence of vibration There is a difference. At the same time, the output waveforms of different vibration events are different, and the output waveforms of similar vibration events are similar. As shown in Figure 8 。

Because the dual MZ interference technology to monitor fiber vibration has the characteristic of "different types of vibration events have different output waveforms, and the output waveforms of similar vibration events are similar" [13], this technology has the ability to identify the types of vibration events and avoid $\Phi$ - The OTDR technology monitors defects with a high rate of vibration false alarms, and is more suitable for long-term monitoring of optical fiber vibration to determine the degree of damage caused by external vibration events.
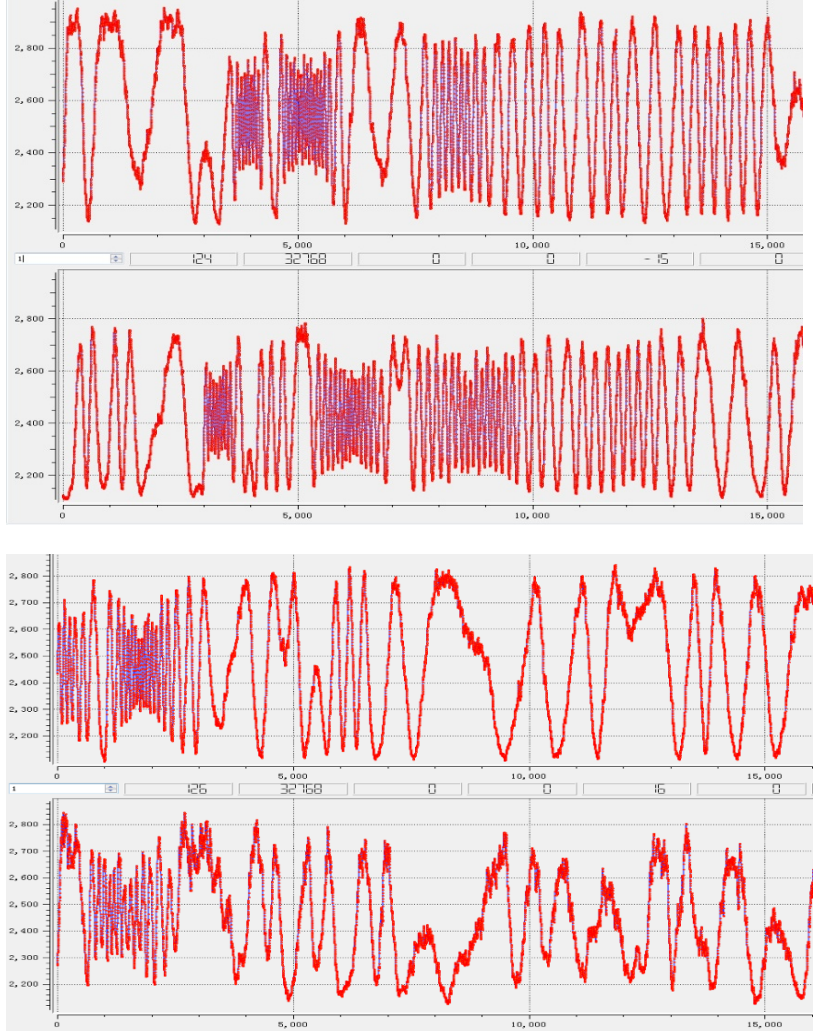

Fig.8. The output waveform of two touch sensor fibers at different times.

\section{Use optical fiber vibration sensor to determine the degree of damage caused by external force intrusion}

If there is an external force intrusion event that may endanger the safety of the cable trench, such as road excavation and other events, before it causes actual damage, the generated vibration will inevitably be transmitted to the cable trench and be captured by the sensing fiber. Using this principle, the optical fiber vibration sensor detects the intrusion of external forces early and determines the degree of damage. External force intrusion hazards can be classified into the following three categories:

(1) Non-threatening external events

When the output waveform of the optical fiber vibration sensor is similar to Figure 5, it can be determined that there is no external force event that can threaten the safety of the optical fiber and the cable trench. At this time, the source of the vibration of the sensing fiber may be the background noise of the natural environment, heavy vehicles passing the road directly above the cable trench, and construction projects far away from the cable trench. In short, such events do not threaten the safety of the cable.

(2)Direct contact with optical cable incident

When the output waveform of the optical fiber vibration sensor is similar to that shown in Figure 6, it can be determined that someone directly touched the optical cable, including the hand or other parts of the body, or touched the optical cable with a stick or other 
object. This shows that someone has invaded the cable trench and should be taken seriously and checked.

(3)Threatening incidents of external force violations

When the output waveform of the optical fiber vibration sensor is similar to Figure 7, it can be determined that there is excavation near the sensing fiber, including large excavator construction and small manual excavation, and the construction site is very close to the cable trench where the sensing fiber is located, which is enough to threaten Cable safety. It should be taken seriously and verified.

The judgment of the event represented by the output waveform will be automatically completed by the optical fiber vibration sensor ${ }^{[14]}$. When the optical fiber vibration sensor judges that the output waveform belongs to (2) (3) events, the machine automatically outputs prompt information, which includes event type.

\section{A new way of cable operation and maintenance inspection}

\subsection{Traditional underground cable inspection method}

This time, the discussion on cable operation and maintenance inspection methods is aimed at buried cables. In order to protect the cables from deliberate damage by construction and personnel, the power unit needs to patrol and inspect the cables and discover incidents and behaviors that may damage the optical cables. Traditional underground cable inspection methods ${ }^{[15]}$ mainly include the following items:

(1) Manual periodic inspection

The personnel of the power grid or operation and maintenance unit regularly (usually once a week) inspect all the cable trench lines in the jurisdiction. The first is to inspect whether there is construction on the ground along the way of the cable trench, especially large-scale mechanical construction; the second is to check whether the cable duct is normal; the third is to check whether the cable joints on the key nodes are abnormal. The following problems exist in regular manpower inspections:

First of all, the effect of purely human inspection depends on the subjective initiative of inspectors, and this kind of work is highly repetitive and complicated in content. There are many uncertain factors in its effect and lack of guarantee. During the inspection process, there is a greater possibility of missing or missed inspection;

Secondly, there is a window period for regular manpower inspections (usually about one week). Construction projects implemented during the empty window period, especially some illegal constructions carried out secretly, cannot be subject to the timely and effective supervision of manual inspections.

(2) Establish a communication platform with the construction unit

Establish a communication platform (such as establishing a WeChat group, etc.) between the power

grid department and the responsible personnel of the main construction units in the jurisdiction. The construction unit communicates with the person in charge of the power grid through the communication platform when carrying out the project. If the construction project is near the buried cable and requires the use of large-scale excavating machinery, the power grid will send staff to supervise the site. This method depends on the degree of subjective cooperation of the construction party. The following problems exist in the way of establishing a communication platform:

First of all, when the project construction area is close to buried cables, the power grid will require it not to use large-scale machinery and must switch to manual construction. For the construction unit, this seriously affects the construction efficiency and is not in line with its intuitive interests. Due to luck, the construction unit may conceal the project.

Secondly, the main participants in the exchange platform are government departments and large construction companies. Due to the reasons stated in a., some small construction units do not participate in the exchange platform. Therefore, this method lacks communication and supervision of such construction units.

(3) Mutual notifications within the power grid

After the power grid personnel discover the construction project, internally notify the management personnel of the corresponding jurisdiction. This method can make up for the empty window period of manpower regular inspections to a relatively small extent. The main problem of the way of mutual notification between the power grids is that there are great limitations and accidents, which can only be used as a supplement to the above two inspection methods.

To sum up, the traditional underground cable inspection method mainly relies on manpower, and the inspection effect is also extremely dependent on the subjective initiative of the inspection participants. Therefore, the inspection effect has many uncertain factors such as many blind spots and window periods.

\subsection{A new underground cable inspection method combined with optical fiber vibration sensing technology}

Combining the technology described in Chapter 3 to determine the degree of damage to optical cables by external forces using optical fiber vibration sensing technology, a new cable inspection method is proposed here. The main content includes the following items:

(1) Use the optical fiber vibration sensor to construct a distributed optical fiber vibration warning system by using the power communication optical cable laid in the same trench as the cable to monitor the vibration along the cable trench where the optical cable is located in real time. 9:

The system hardware composition is shown in Figure 


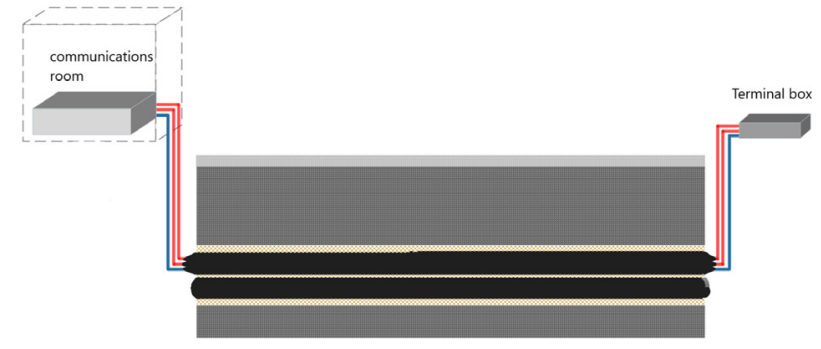

Fig.9. Hardware composition of distributed optical fiber vibration early warning system.

The optical fiber vibration sensor is installed in the communication room, and is connected to the three spare optical fibers of the communication optical cable, and the terminal is connected to a dedicated optical terminal box. This constitutes the hardware part of the distributed optical fiber vibration early warning system. All external force events that threaten the safety of optical cables and cables, the vibrations of which will be transmitted to the optical cables first, which will be detected by the distributed optical fiber vibration early warning system, and an early warning will be issued before it actually causes damage. The system not only protects the optical cable, but also protects the cable trench and cable part through which the optical cable passes.

(2) Build a software system for global vibration monitoring of optical cables in the form of GIS. The system can automatically identify external force hazards that threaten the safety of optical cables and issue warnings by means of map positioning. The software system is the software part of the distributed optical fiber vibration early warning system.

The software system displays the GIS routing of the communication optical cable, and performs $7 \times 24$ hours real-time monitoring and protection of the line. The optical fiber vibration sensor receives the signal of the optical fiber vibration, and first screens out the parts that are threatening to the optical cable and the cable. After that, the system converts the location information of this part of the vibration event into GIS positioning, visually presents it in the interface, and displays information such as event type and occurrence time.

(3) Establish a new type of cable inspection mechanism that cooperates with a distributed optical fiber vibration early warning system and manual inspection:

1)The system of "establishing a communication platform with the construction unit" and "mutual notification within the power grid" remains unchanged;

2)The frequency of manual periodic inspections can be reduced, such as once or twice a month;

3)The distributed optical fiber vibration early warning system operates $7 * 24$ hours without interruption. When an external force event that threatens the safety of the cable occurs, such as excavation construction and deliberate sabotage by personnel, the early warning system will immediately and automatically send early warning information to the inspector closest to the scene or in the area where the event is in charge, including the type and location of the event, Instruct inspectors to rush to the scene to deal with external intrusions;
4)Regularly assign personnel to maintain the optical fiber vibration sensor in the computer room.

\subsection{Advantages of the new underground cable inspection method}

The buried cable inspection method combined with optical fiber vibration sensing technology can realize the prevention of external damage to the urban power grid communication optical cables (cables laid in the same trench) and channels, and the controllability and manageability of routing resources, thereby reducing the occurrence of power cables The probability of serious failures reduces economic losses and achieves the effect of obtaining direct economic benefits.

The new buried cable inspection method can effectively supplement the inspection efforts of the inspection mechanism of the urban power communication optical cables (cables laid in the same trench) and the channel to prevent damage by external forces. And greatly improve the efficiency of inspection and maintenance work, realize the effective protection of cables and passages from damage to the cables and passages $7 * 24$ hours, reduce economic losses, and save personnel and maintenance costs. Supplement to the inspection mechanism and efficiency improvement, including but not limited to the following:

(1) The protection of earth-covered cables and passages has changed from regular inspections to realtime monitoring and protection early warning.

(2) Special inspections for abnormal weather have become real-time monitoring and protection early warning.

(3) All-weather, $7 * 24$-hour safety protection for cables laid in the same trench.

(4) Provide a power optical cable monitoring and early warning platform to realize the visual management of physical routing resources of communication optical cables, and realize the manageability and controllability of optical cable resources.

Therefore, it can be expected that the direct economic benefits brought about by the inspection mechanism will be very considerable. At the same time, the new inspection method can improve the reliability and stability of the power supply and signal dispatching of the urban power grid to a certain extent, and the satisfaction and public opinion reputation of high-quality public services in the society, and achieve a win-win situation for enterprises, governments, and citizens. Obtain indirect economic benefits and corporate brand reputation.

\section{Conclusion}

The optical fiber vibration sensing technology using the principle of double $\mathrm{M}-\mathrm{Z}$ interference overcomes the problem of high false alarm rate of general optical fiber vibration sensing technology, while achieving high detection rate and low false alarm rate, which is suitable for long-term monitoring of threatening external force intrusion events. 
Using the communication optical cable laid in the same trench as the cable, the optical fiber vibration sensing technology can be applied to the protection of the cable against damage by external forces. By sensing the vibration of a destructive external force intrusion event, a distributed optical fiber vibration early warning system using optical fiber vibration sensing technology can detect threats in advance. By combining traditional methods such as distributed optical fiber vibration sensing systems and manual inspections, a new type of cable inspection mechanism is constructed. The greatest significance is to achieve real-time full-coverage monitoring of the cable, and to prevent the intrusion of external forces before the cable is actually damaged., To protect business units from losses.

\section{References}

1. YANG Jian. Analysis of $10 \mathrm{kV}$ Power Cable Construction Faults and Preventive Measures[J]. Technological Development of Enterprise, 2015, 34(35):96.

2. YAN Zhiqi. Application of optical fiber communication in power communication[J]. Science \& Technology Information, 2019, 35:5.

3. MA Li. Research on the Vibration Sensing Technology Based on $\varphi$-OTDR[D]. Baoding: North China Electric Power University, 2015.

4. XU Chengjin. Research on Signal Processing Technology of Distributed Optical Fiber Sensing System[D]. Hangzhou: Zhejiang University, 2017:18-19.

5. ZHANG Zhijuan, GUO Wenhan, Xu Zhiniu, et al. Principle and application status of optical fiber sensing technology based on $\Phi-\mathrm{OTDR}[\mathrm{J}]$. Semiconductor Optoelectronics, 2019, 40(1):16.

6. PAN Yue, WANG Jian. Study on location performance of dual Mach-Zehnder interferometer[J]. Optical Instruments, 2012, 34(3):55.

7. HU Guangqiang. Studying distributed optical fiber perimeter and its location technology based on Dual Mach-Zehnder[D]. Beijing: Beijing Jiaotong University, 2017:17-18.

8. ZHAO Hao. Research of fiber vibration sensor system of digital network[J]. Electronic Design Engineering, 2014(19):20.

9. GUAN Guoguang. Time-frequency Analysis for Distributed Optical Fiber Sensing Based on MZ Interferometry and $\Phi-O T D R[D]$. Chengdu: Southwest Jiaotong University, 2016:16-17.

10. SHEN Huan, BAI Yuantao, LIU Jihong. Hierarchical cross-correlation location method for dual $\mathrm{M}-\mathrm{Z}$ optical fiber vibration sensors[J]. Optical Communication Technology, 2019,43,(6):2-3.

11. XIE Weilin. Research on Dynamic Laser Coherence Control Theory, Technologies, and Applications[D]. Shanghai: Shanghai Jiao Tong University, 2017:1-6.
12. REN Jianying, SUN Huayan, ZHAO Yanzhong, et al. Analysis of influencing factors of difference frequency signal of dual frequency laser coherent detection $[\mathrm{J}]$. LASER\&INFRARED, 2020,50(4):408-410.

13. ZOU Baixian, XU Shaowu, MIAO Jun, et al. Feature Extraction and Recognition of Vibration Signals in Optical Fiber Security System[J]. Journal of Computer Research and Development, 2019,56(9):1859-1871.

14. SUN Hui. Research on Signal Recognition Technology in Optical Fiber Security System[D]. Beijing: Beijing University of Posts and Telecommunications, 2019:23-32.

15. NA Ying. Electric cable of electric company of Shenyang perates and maintains the management mode to study[D]. Changchun: Jilin University, 2009:7. 